\title{
Intradermal air pouch leukocytosis as an in vivo test for nanoparticles
}

This article was published in the following Dove Press journal:

International Journal of Nanomedicine

12 December 2013

Number of times this article has been viewed

\author{
Jennifer Vandooren' \\ Nele Berghmans' \\ Chris Dillen' \\ Ilse Van Aelst' \\ Isabelle Ronsse' \\ Liron Limor Israel $^{2}$ \\ Ina Rosenberger ${ }^{3}$ \\ Jörg Kreuter ${ }^{3}$ \\ Jean-Paul Lellouche ${ }^{2}$ \\ Shulamit Michaeli ${ }^{4}$ \\ Erica Locatelli ${ }^{5}$ \\ Mauro Comes Franchini ${ }^{5}$ \\ Miren K Aiertza ${ }^{6}$ \\ Laura Sánchez-Abella ${ }^{6}$ \\ Iraida Loinaz ${ }^{6}$ \\ Dylan R Edwards ${ }^{7}$ \\ Louis Shenkman ${ }^{8}$ \\ Ghislain Opdenakker
}

'Rega Institute for Medical Research, University of Leuven, Leuven, Belgium; ${ }^{2}$ Department of Chemistry and Institute of Nanotechnology and Advanced Materials, Bar-llan University, Ramat Gan, Tel Aviv, Israel; ${ }^{3}$ Institut für Pharmazeutische Technologie, Johann Wolfgang GoetheUniversität, Frankfurt, Germany; ${ }^{4}$ The Mina and Everard Goodman Faculty of Life Sciences and Advanced Materials and Nanotechnology Institute, Bar-llan University, Ramat Gan, Tel Aviv, Israel; ${ }^{5}$ Department of Industrial Chemistry Toso Montanari, University of Bologna, Bologna, Italy; ${ }^{6} \mathrm{New}$ Materials Department, Fundación CIDETEC, San Sebastián, Spain; ${ }^{7}$ School of Biological Sciences, University of East Anglia, Norwich, UK; ${ }^{8} \mathrm{Tel}$ Aviv University, Ramat Aviv, Tel Aviv, Israel

Correspondence: Ghislain Opdenakker Rega Institute for Medical Research, KU Leuven, Minderbroedersstraat 10 B-3000, Leuven, Belgium

Email ghislain.opdenakker@rega.kuleuven.be
Abstract: The need for test systems for nanoparticle biocompatibility, toxicity, and inflammatory or adaptive immunological responses is paramount. Nanoparticles should be free of microbiological and chemical contaminants, and devoid of toxicity. Nevertheless, in the absence of contamination, these particles may still induce undesired immunological effects in vivo, such as enhanced autoimmunity, hypersensitivity reactions, and fibrosis. Here we show that artificial particles of specific sizes affect immune cell recruitment as tested in a dermal air pouch model in mice. In addition, we demonstrate that the composition of nanoparticles may influence immune cell recruitment in vivo. Aside from biophysical characterizations in terms of hydrodynamic diameter, zeta potential, concentration, and atomic concentration of metals, we show that - after first-line in vitro assays - characterization of cellular and molecular effects by dermal air pouch analysis is straightforward and should be included in the quality control of nanoparticles. We demonstrate this for innate immunological effects such as neutrophil recruitment and the production of immune-modulating matrix metalloproteases such as MMP-9; we propose the use of air pouch leukocytosis analysis as a future standard assay.

Keywords: nanoparticles, biocompatibility, toxicity, air pouch, immunology

\section{Introduction}

Since its inception more than 55 years ago, the air pouch model has been linked with cancer research. Hans Selye used the model to study the controversial and contested role of inflammation in tumor development and growth. He generated a proximal and caudal air pouch on dorsal sites in rats and injected these with Walker tumor cell suspensions in combination with irritants or immune suppressants. ${ }^{1}$ From these experiments, he concluded that "a topical inflammatory response can greatly enhance the development of transplanted neoplastic tissue," forecasting that specific inflammatory reactions constitute key driver activities in oncogenic processes. ${ }^{2}$ Subsequently, Selye's granuloma pouch model was used to study other forms of immunomodulation, for example, by malnutrition and other noxae. ${ }^{3}$ Another historical example of air pouch use was the isolation of a tumor angiogenesis factor (TAF) from Walker tumor-elicited ascitic fluid and the demonstration of its angiogenic effect in the rat dorsal air sac assay. ${ }^{4}$

The first use of the air pouch model to study infection and inflammation was also a half century ago. ${ }^{5}$ On the basis of in-depth histopathological studies, it was concluded that air pouch formation and the infiltration of leukocytes are dynamic processes in need of standardization. The air pouch lining development, including formation of blood vessels and a mechanical barrier to retain products, was found to be ideal at 6 days, 
and cell infiltration was optimal at 24 hours after product injection. ${ }^{6}$ For a considerable time, the rodent air pouch model was assumed to reflect the clinical situation of healing wound cavities after extirpation of organs. ${ }^{7}$ Thereafter came a period when it was recognized that this model is useful for the study of immune reactions, bone and cartilage breakdown, and also for drug testing. ${ }^{8-13}$ In addition to cell infiltrations, the production of biological agents, eg, matrix metalloproteinases (MMPs), was studied in the pouch exudates. ${ }^{14}$ Rats were used in most of these studies, but these were gradually replaced by mice, coincidently with the explosion of genetic and immunological studies using mice for in vivo testing. ${ }^{15}$ Air pouch models were used to study mechanisms of action of drugs, pharmacokinetics, enzyme induction or inhibition, regional drug delivery, angiogenesis, and cell migration. ${ }^{7,14,16-22}$ Gradually, the mouse air pouch model became a standard test in pharmacological, immunological, and biomaterials research. ${ }^{23-27}$ With the application of the mouse air pouch model, genetic factors, including those encoding cytokines, proteinases and other enzymes, and adhesion molecules were identified in inflammation; the combination with spontaneous gene deficiencies or gene knockout technology further enhanced insights into inflammatory processes. ${ }^{28-33}$

With the introduction of nanomedicines, the awareness of immunomodulating effects has increased but still remains problematic and challenging. Laboratories are collaborating worldwide to define, compare, and validate, not only toxicology tests, but also assays to measure effects of nanoparticles on immune responses. ${ }^{34}$ At present, in vivo and ex vivo immunological analysis of injected nanoparticles is done by studying white blood cells and the histopathology of immune organs. Blood analysis yields a partial picture, and histopathology is time consuming and not quantitative. For immunological screening of nanomedicines, these tests are important but not sufficient - for at least three reasons. First, all viruses, except the largest (pox and pandora) viruses, are (replicating) nanostructures that exert profound immunological effects in vivo, even after being completely inactivated, for instance, in vaccines. Nanoparticles, in particular those that are (un) purposely coated with proteins or those having nucleic acids, are therefore virus mimetics. It is generally accepted that pattern recognition receptors, such as Toll-like or retinoic acid-inducible gene (RIG)-like receptors, induce the immediate innate immune response to nanoparticulate viruses. Nanoparticles also help with ensuing adaptive responses to generate antigen-specific $\mathrm{T}$ cell responses and antibody formation by B lymphocytes. In addition, specific cell types in organisms can sense the size of particles (infectious agents range in size from nanometers for viruses, micrometers for bacteria and parasites, to millimeters, centimeters, or even meters for worms). Second, host immunity is by definition based on organ systems that cannot be replaced by single cells. For instance, antigenicity is tested by the ability to form antibodies in vivo; such a test is difficult to reproduce in vitro. Third, the available in vivo toxicity tests for nanoparticles include skin painting, inhalation (airway provocation), and ingestion. These tests are excellent means by which to establish safety measures against potential dangers of professional exposure of workers in research and manufacturing. However, in all the aforementioned cases, epithelial barriers of skin and mucosa are involved. Most nanomedicines will, in practice, be used by injection and therefore will first encounter leukocytes, and endothelial and mesothelial cells, rather than epithelial cells. For these reasons, a profound need exists for one or more standard tests that can measure various aspects of immunological responses. In view of increasing biomedical applications of nanoparticles as drugs or imaging tools, immunotoxicity analysis needs standardization. ${ }^{35,36}$ In this study, we present data on the use of air pouch analysis of nanoparticles toward this goal.

\section{Materials and methods}

\section{The mouse air pouch model}

A general outline of the method used is shown in Figure 1A. Dermal air pouches were established by injecting Naval Medical Research Institute mice on dorsal sites with $3 \mathrm{~mL}$ filtered air $(0.20 \mu \mathrm{m}$ filter $)$ on days 0 and $3 .{ }^{37}$ On day 6 , the test samples were injected, along with a negative and a positive control sample. Phosphate-buffered saline (PBS) was used as a negative control and chlorite-oxidized oxyamylose (COAM) as a positive control, for neutrophil recruitment. ${ }^{38}$ After 24 hour contact, the exudates of the pouches were collected after injection of $1 \mathrm{~mL}$ pyrogen-free PBS containing heparin $20 \mathrm{U} / \mathrm{mL}$ and $2 \%$ fetal calf serum (FCS), and followed by 30 seconds of gentle massage. This procedure was repeated twice with $2 \mathrm{~mL}$ buffer, and the total exudate collection was centrifuged (10 minutes, $900 \mathrm{rpm}$ ). The numbers of viable cells were determined in a Bürker chamber after trypan blue exclusion of dead cells and were resuspended for immediate hemocytometry and cytospin analysis. The supernatant fluids were frozen $\left(-20^{\circ} \mathrm{C}\right)$ for gelatin zymography. ${ }^{39}$ Each sample was tested for contamination with endotoxin using the Limulus amebocyte lysate test (Cambrex Bio Science, Baltimore, MD, USA). All procedures were conducted in accordance with protocols approved by the local Ethics Committee (Licence number P028/2013, Belgium). All 
A
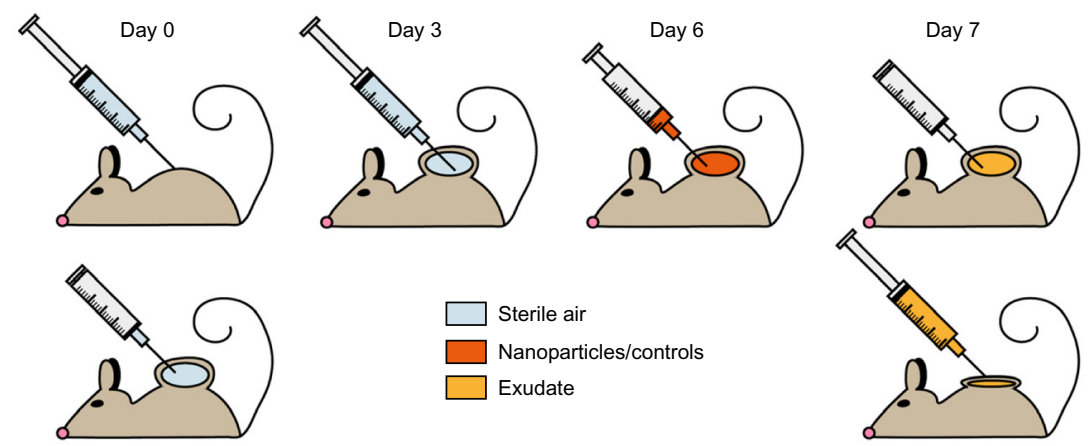

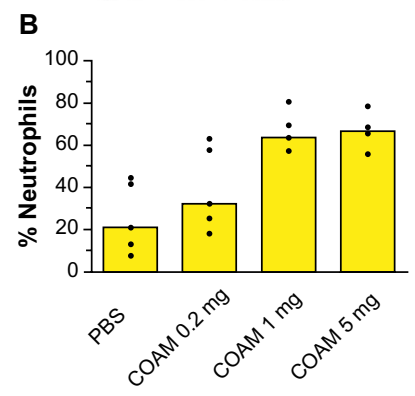

C

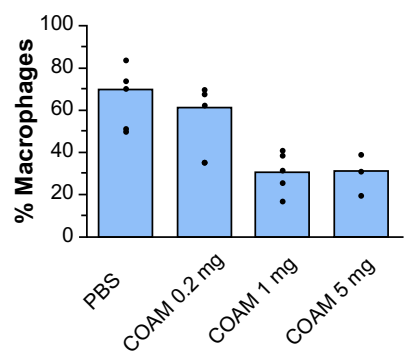

D
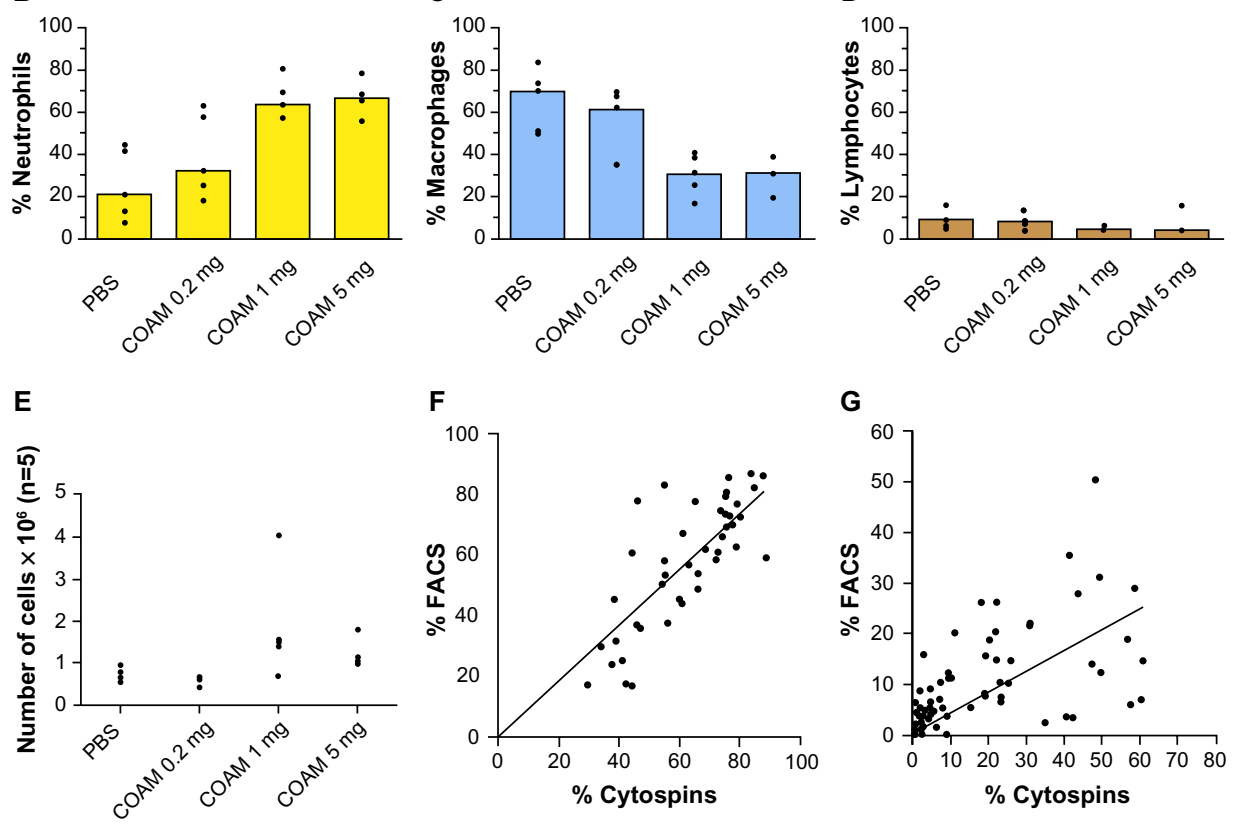

$\mathbf{F}$

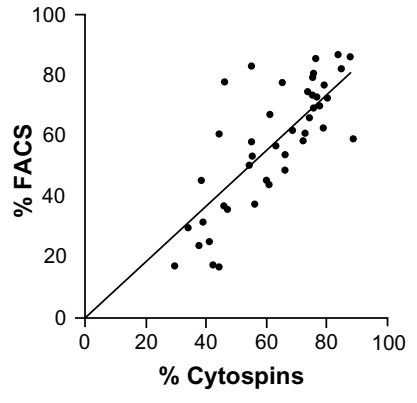

G

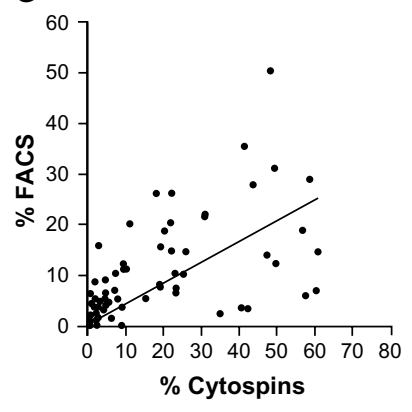

Figure I Optimization of the air pouch model.

Notes: (A) Schematic outline of the air pouch model. At the start of the test, $3 \mathrm{~mL}$ air is injected through a sterile $0.20 \mu \mathrm{m}$ filter at a dermal site of a mouse to form a pouch. On the third day, this action is repeated. Products are injected on day 6 after the start and their cellular and molecular effects are measured 24 hours later by retrieval of cells and molecules within the pouch exudates. (B-D) COAM induces a dose-dependent recruitment of neutrophils and can be used as a standard for the air pouch model. The air pouch model was executed in the standard setting of 6 day formation and 24 hour contact time for test samples. COAM was used at different doses. Three possible read-outs are shown: (B) increase (and saturation) of percentage neutrophils, (C) decrease of macrophage percentages and (D) decrease of percentage lymphocytes ( $\mathrm{n}=5$ for all test samples). The positive control reached saturation at I mg COAM per pouch. (E) Analysis of the absolute numbers of retrieved cells per pouch. (F and $\mathbf{G}$ ) Correlation between FACS and cytospin data from mouse air pouches. (F) Correlation (two-tailed parametric correlation analysis) between percentages of neutrophils (polymorphonuclear cells) as measured by FACS analysis and cytospin analysis $(r=0.80, n=45)$. Linear regression was performed and found to be significant $(P<0.000 \mathrm{I})$. (G) Correlation (two-tailed parametric correlation analysis) of percentages mononuclear cells as measured by FACS analysis and cytospin analysis ( $r=0.58$, $n=79$ ). Linear regression was performed and found to be significant $(P<0.000 \mathrm{I})$.

Abbreviations: COAM, chlorite-oxidized oxyamylose; FACS, fluorescence-activated cell sorting; PBS, phosphate-buffered saline.

materials were tested on three to five mice. In addition, the statistical analysis and the repeat numbers of the positive (COAM) and negative (PBS) controls have been indicated in Table 1. Finally, most nanoparticles were tested twice (with similar results), depending on material availability.

\section{Differential cell counts}

Differential cell counts were based on morphologic examination of cytospin preparations stained with Hemacolor (Merck Chemicals, Darmstadt, Germany). $75 \times 10^{3}$ cells were applied onto slides by centrifugation using a Shandon Cytospin 2 apparatus (Thermo Shandon, Pittsburgh, PA, USA).

\section{Flow cytometry analysis}

To corroborate hemocytometric analyses, we also performed flow cytometry analysis. The rationale for this was two-fold. First, many laboratories are equipped with cytometers to differentiate by immunophenotyping cell numbers in an efficient way. Second, it was used to validate the more labor-intensive microscopic quantification. We incubated $5 \times 10^{5}$ cells for 15 minutes with the Fc-receptor-blocking antibodies anti- 
Table I The effect of different sets of nanoparticles according to the air pouch test

\begin{tabular}{|c|c|c|c|c|}
\hline Production & Nanoparticle ${ }^{t t t}$ & LAL assay (pg/mg) & Cells $\left(\times 10^{6}\right)$ per air pouch & \% Neutrophils \\
\hline & PBS & $\leq 12.5^{*}$ & $0.77 \pm 0.39^{\dagger}$ & $37.8 \pm 6.9^{\dagger+}$ \\
\hline & COAM & 13.3 & $\mathrm{I} .53 \pm 0.5 \mathrm{I}^{\dagger}$ & $60.7 \pm 7.7^{\text {t十 }}$ \\
\hline BIU, Israel & CANsmaghemite NPs ${ }^{1 a}$ & 28.4 & 2.77 & 76 \\
\hline BIU, Israel & Type I PEI-CAN-maghemite ${ }^{\mathrm{lb}}$ & $\leq 9.3$ & 0.91 & 88 \\
\hline BIU, Israel & Type II PEI-CAN-maghemite ${ }^{l c}$ & 42.5 & 2.83 & 55 \\
\hline CIDETEC, Spain & PDMAEMA-SCPNs ${ }^{2 a}$ & 562.5 & 1.75 & 89 \\
\hline CIDETEC, Spain & PMAAc-SCPNs ${ }^{2 b}$ & 762.5 & 0.45 & 56 \\
\hline UNIBO, Italy & PLGA-COOH ${ }^{3 a}$ & $\leq 3.1$ & 0.9 & 34 \\
\hline UNIBO, Italy & PLGA-b-PEG-COOH ${ }^{3 b}$ & 12.5 & 1.1 & 39 \\
\hline UNIBO, Italy & Magh@PNPs ${ }^{3 c}$ & 41.7 & 1.22 & 44 \\
\hline GU, Germany & LNP LII ${ }^{4 a}$ & 12 & 0.89 & 59 \\
\hline GU, Germany & CAN CIII' & $\leq 1.3$ & 0.74 & 39 \\
\hline
\end{tabular}

Notes: ${ }^{\dagger}$ The amount of cells per air pouch in the PBS and COAM conditions was calculated over a set of seven experiments with three mice per condition. The values are shown as mean \pm SD. The PBS and COAM groups were significantly different as calculated with a paired $t$-test $(n=7, P=0.0043)$. ${ }^{\dagger T}$ The percentage of neutrophils per air pouch in the PBS and COAM conditions were calculated over a set of seven experiments with three mice per condition. The values are shown as mean \pm SD. The PBS and COAM groups were significantly different as calculated with a paired $t$-test $(n=7, P=0.0032)$. ${ }^{+t+} \mid$ dentification and characteristics of nanoparticles: Ia-c: $(I a) C A N-\gamma$-Fe ${ }_{2} \mathrm{O}_{3} N P s$ : average NP diameter 7.6I $\pm 2.33 \mathrm{~nm}$ (TEM), weight ratio Ce/Fe: 0.029; (Ib) Type I PEI ${ }_{25}-\mathrm{CAN}-\gamma-\mathrm{Fe}_{2} \mathrm{O}_{3} \mathrm{NPs}$ average NP diameter 6.50 $\pm 2.15 \mathrm{~nm}$ (TEM), $7 \mathrm{I} .2-96.56 \mathrm{~nm}$ (DLS average hydrodynamic NP diameter), $\zeta$ potential: $+23.0-24.0 \mathrm{mV}$; weight ratio Ce/Fe: 0.095; (Ic) Type II PEI $-\mathrm{CAN}_{8} \gamma-\mathrm{Fe}_{2} \mathrm{O}_{3} \mathrm{NPs}$ : average NP diameter 7.65 $\pm 2.64 \mathrm{~nm}$ (TEM), 58.0-62.0 nm (DLS average hydrodynamic NP diameter), $\zeta$ potential: $+56.3 \mathrm{mV}$; weight ratio Ce/Fe: 0.100. 2a and b: (2a) PDMAEMA-SCPNs: N,N'-dimethylaminoethyl methacrylate-based single chain polymer nanoparticles, $\pm 13 \mathrm{~nm}(\mathrm{DLS}), \zeta$ potential: $+1 \mathrm{I} .5,(2 \mathrm{~b})$ PMAAc based SCPNs: polymethacrylic acid-based single chain polymer nanoparticles, \pm 17 nm (DLS); 3a-c: (3a) PLGA-COOH: average NP diameter $160 \mathrm{~nm}$, $\zeta$ potential: -55.I mV, PDI: 0.078, (3b) PLGA-b-PEG-COOH: average NP diameter $74 \mathrm{~nm}, \zeta$ potential: - II $.5 \mathrm{mV}$, PDI: 0.095; (3c) Magh@PNPs: average NP diameter $92.3 \mathrm{~nm}$, $\zeta$ potential: $-7.56 \mathrm{mV}$, PDI: 0.098 . 4a and b: (4a) LNP LIl: average NP diameter $193 \pm$ I nm, $\zeta$ potential: $-41.9 \pm 0.7 \mathrm{mV}$, PDI: $0.023 \pm 0.02$ I, (4b) CAN CIII: average NP diameter I $9 \mathrm{I} \pm$ I nm, $\zeta$ potential: $-52.7 \pm 0.3 \mathrm{mV}$, PDI: $0.027 \pm 0.035$. *For PBS the endotoxin analysis was obtained per $\mathrm{mL}$ volume. For all other products we calculated the endotoxin level/mg pure product.

Abbreviations: BIU, Bar-llan University; CAN, ceric ammonium nitrate; CIDETEC, Fundación CIDETEC; COAM, chlorite-oxidized oxyamylose; DLS, dynamic light scattering; GU, Goethe-Universität; LAL, Limulus amebocyte lysate; LNP, lipid nanoparticle; Magh, Maghemite; n, number; NP, nanoparticle; PBS, phosphate-buffered saline; PDI, polydispersity index; PDMAEMA, poly(N,N-dimethylaminoethyl methacrylate); PEG, polyethylene glycol; PEI, polyethyleneimine; PLGA, poly(D,L-lactide-co-glycolide); PMAAc, polymethacrylic acid; PNP, polymeric nanoparticles; SCPN, single-chain polymeric nanoparticle; TEM, transmission electron microscopy; UNIBO, University of Bologna; SD, standard deviation.

CD16/anti-CD32 (BD Biosciences Pharmingen, San Diego, CA, USA), washed with PBS $+2 \%$ FCS and then stained for 30 minutes with the indicated fluorescein isothiocyanate (FITC)-conjugated, phycoerythrin (PE)-conjugated and allophycocyanin (APC)-conjugated antibodies. Cells were washed twice and fixed with $0.37 \%$ formaldehyde in PBS. FITC-conjugated anti-CD11b, PE-conjugated anti-CD4, FITC-CD8, PE-conjugated Gr-1 (granulocyte-differentiation antigen-1), and APC-conjugated F4/80 were purchased from eBioscience, Inc (San Diego, CA, USA). Cells were analyzed on a fluorescence-activated cell sorting (FACS) Calibur flow cytometer (Becton Dickinson, San Jose, CA, USA) and data were processed with the manufacturer's CellQuest software. $^{38}$

\section{Gelatin zymography}

Gelatin zymography was used to measure the presence of the MMP-2 and the inducible MMP-9..$^{39,40}$ All samples were first prepurified by gelatin-Sepharose affinity chromatography, as described in a previous publication. ${ }^{41} \mathrm{Next}$, the proteins were separated in $7.5 \%$ polyacrylamide gels to which $0.1 \%$ gelatin (Sigma-Aldrich, St Louis, MO, USA) was added and copolymerized. After electrophoresis, the gels were washed to remove sodium dodecyl sulfate and incubated for the development of zymolytic bands. The gels were stained with Coomassie brilliant blue R-250 (Sigma-Aldrich).

\section{Results}

\section{Validation of the mouse air pouch model}

First, the use of COAM as a positive control substance was evaluated (Figure 1B-E). Injection of COAM resulted in a dose-dependent increase in the number of total viable cells. Differential cell counts revealed that mostly neutrophils migrated into air pouches. Therefore, in all forthcoming experiments $2 \mathrm{mg}$ of COAM was used as a positive control for neutrophil recruitment. Upon collection of the samples, we first counted and differentiated the cells by classical hemocytometric analysis. With this approach a trained microscopist can differentiate neutrophils from monocytes/macrophages and lymphocytes, and this technique does not necessitate the purchase of expensive equipment. To validate this approach and to demonstrate that, alternatively, flow cytometric analysis with the use of cell-specific antibodies is another way of quantification, correlation analysis was done for neutrophil and mononuclear cell counts for both techniques (Figure 1F and $G$ ). Our data indicate that both methods are suitable for 
quantification and differential cell counting of specific leukocyte subsets, and that more sophisticated and high-end cell differentiations with flow cytometric analysis are possible.

\section{Analysis of differently sized polystyrene particles}

In a first set of experiments we evaluated the effect of polystyrene particles of different sizes on the recruitment of leukocytes and the content of gelatinases (MMP-2 and MMP-9). Because MMP-9 is highly expressed by leukocytes upon an inflammatory stimulus, we hypothesized that immunemodulating substances would increase the ratio of MMP-9/ MMP-2. ${ }^{40,42}$ Indeed, in gelatin zymography analysis, ${ }^{39}$ MMP-2 is found constitutively expressed in most tissues and body fluids. Consequently, MMP-2 may be used as an internal reference enzyme. In contrast, MMP-9 is an inducible enzyme in cancer, infection, and inflammation, which makes the MMP-9/MMP-2 ratios indicative for neoplastic, infectious, or inflammatory processes.

Whereas COAM and polystyrene nanoparticles induced more than a doubling in the numbers of total retrieved cells in comparison with PBS control samples, the microspheres did not (Figure 2A). When we differentially counted leukocytes in this pilot experiment, we observed twice as many neutrophils after nanoparticle and microparticle injection than with the PBS control, which recruited more mononuclear cells (Figure 2B and C). Zymography analysis of the exudate fluids demonstrated a clear induction of gelatinase B/MMP-9 by COAM and by the polystyrene nanospheres, but less with microspheres. As expected, the cellular infiltration was correlated with the induction of MMP-9; these findings illustrated the inflammatory context of air pouches at the molecular level after injection of COAM (Figure 2D and E).
A

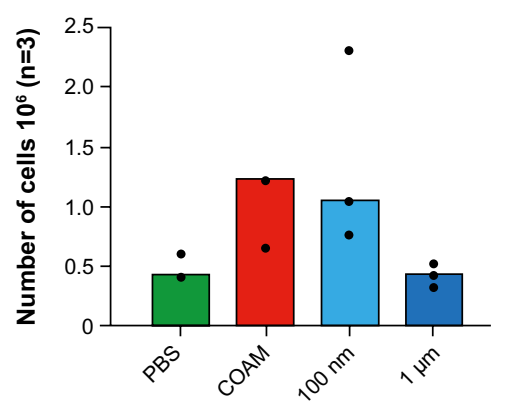

B

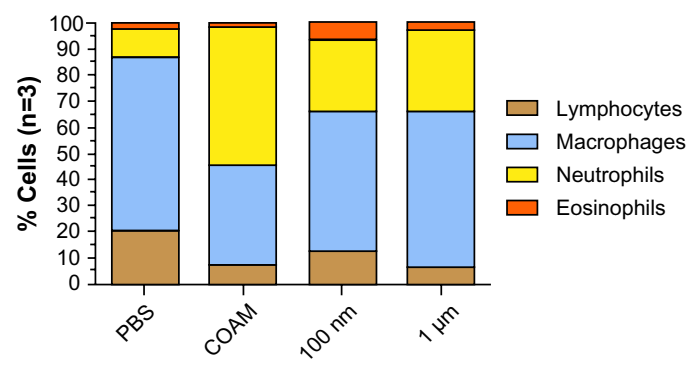

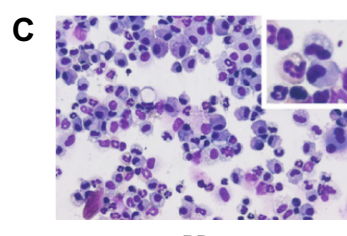

PBS

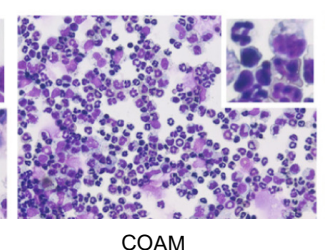

COAM

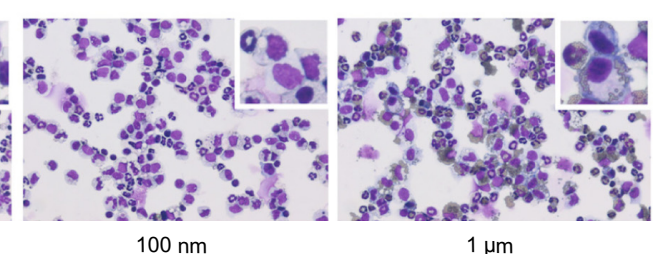

$100 \mathrm{~nm}$
$1 \mu \mathrm{m}$
D

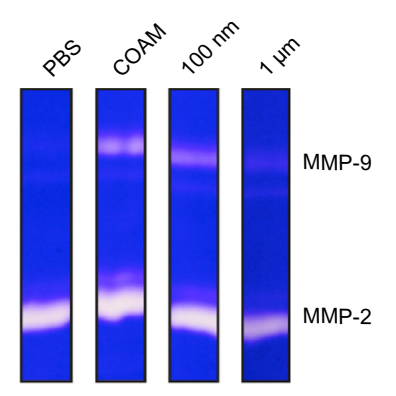

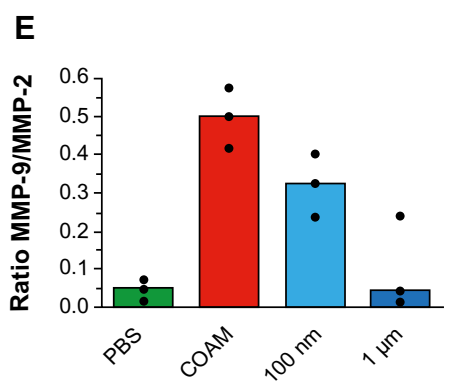

Figure 2 Analysis of polystyrene particles with different sizes in air pouches.

Notes: Particles were either endotoxin-free polystyrene nanoparticles (100 nm) or polystyrene microspheres with a diameter of I $\mu \mathrm{m}$. (A) Analysis of the absolute numbers of retrieved cells. (B) The relative numbers of leukocytes as determined by hemocytometry and as corroborated by FACS analysis. (C) Illustration of cytospin preparations stained with hemacolor and higher magnifications of the cells in the insets. (D) Illustration of gelatin zymography analysis of air pouch fluids. Gelatinase A/ matrix metalloproteinase-2 (MMP-2) is constitutively expressed in all samples and gelatinase B/MMP-9 is up regulated after injection of COAM or polystyrene nanoparticles. (E) Scanning densitometry analysis of gelatin zymograms. The MMP-9 content is expressed as the ratios of MMP-9 versus constitutive MMP-2. The results illustrate the acute inflammatory context of air pouches, after COAM treatment, at the molecular level. Clearly, cellular infiltration is correlated with the induction of MMP-9.

Abbreviations: COAM, chlorite-oxidized oxyamylose; FACS, fluorescence-activated cell sorting; MMP, matrix metalloproteinase; PBS, phosphate-buffered saline. 


\section{Analysis of core nanoparticles}

Sets of different nanoparticles with different chemical characteristics and manufacturing procedures were compared in the dermal air pouch system with negative (PBS) and positive (COAM) control samples (Table 1 and Supplementary materials). First, all samples were tested for contamination with endotoxin. Most particles did not exceed the endotoxin limit of $100 \mathrm{pg} / \mathrm{mL}$ (US Food and Drug Administration standard for substances in direct/indirect contact with the cardiovascular system or lymphatic system). However, several nanoparticles attracted higher percentages of neutrophils, resulting in higher total cell counts in comparison with the negative control.

\section{Discussion}

Most reviews on safety, toxicity and immunological effects of nanoparticles use in vitro analyses. Without any doubt, such tests are paramount and essential first-line analytical tools for the measurement of cytotoxicity and eventual metabolic effects. ${ }^{34-36}$ However, the effects of nanoparticles on in vitro cultured macrophages, dendritic cells, neutrophils, and lymphocytes, in terms of release of inflammatory (eg, prostaglandins) or immunological mediators (eg, enzymes and cytokines) are insufficient to determine immunotoxicity.

The next step, second-line analytical tools for in vivo testing, logically starts with the analysis of blood components. Hematological parameters should include effects on the clotting and fibrinolytic systems, and blood cell counts for erythrocytes (hemolysis), platelets and leukocytes. Immunological parameters, such as complement pathway tests, immunoglobulin levels, and FACS analysis of leukocyte subsets, may be added to immunological tests for nanoparticles. Another second-line immunological test may consist of histopathology analysis of immune organs, including bone marrow, thymus, lymph nodes and spleen. Practically, however, this constitutes a time-consuming and high-end analysis needing the expertise of well-trained pathologists. ${ }^{34-36}$ Therefore, a second-line in vivo test, amenable to standardization and routine analysis is proposed. The air pouch model fulfills the criteria as such a test for several reasons: 1) it constitutes a surrogate test for histopathology analysis of immune reactions including tissue inflammation; ${ }^{6} 2$ ) dependent on the execution and standardization, it yields (semi-) quantitative results in the form of absolute and relative leukocyte counts; 3 ) it can be used to study acute and chronic immune reactions; 4) it can be executed with limited costs and in simple laboratory environments by well-trained technicians, and with basic infrastructure; and 5) it can even be used to test the effects of (immunomodulating) gases, such as $\mathrm{NO}$ or $\mathrm{H}_{2} \mathrm{~S}$, or of nanoparticles that might generate gases by catalysis.

An important issue to address is the minimization of use of mammalian hosts for in vivo testing. Unfortunately, immunological testing requires in vivo experiments, but in practice the air pouch test yields solid and reproducible data with minimally three mice per test sample. Although we propose to use the air pouch leukocytosis analysis as a standard technique for in vivo testing of nanoparticles and suggest using buffer conditions as negative and COAM as positive controls, we acknowledge that additional studies will demonstrate its value. Figure 1B-E illustrates the variability within groups of five mice, whereas the statistical analysis of the PBS and COAM controls in Table 1 (seven groups, three mice per group) illustrates the reproducibility. Many mouse strains are genetically and immunologically well characterized and can be housed in a cost-effective way in comparison with larger mammals. Also, it needs to be stated that with the mouse air pouch model, the distress and suffering of the animals is limited in comparison with efficacy testing of nanoparticles, for instance, in mouse tumor models. As alternative to the air pouch test, we also used intraperitoneal injection. ${ }^{43}$ Because the peritoneal cavity is a larger space, dilution of samples occurs and, in addition, sample retrieval is more variable and demands better skills. The air pouch test can be executed by any well-trained scientist and, in principle, does not need sophisticated equipment. In addition, analysis methods on retrieved cells and exudate molecules can be upgraded to high-end cytometry and molecular analysis, including RNA sequencing, proteomics, and glycomics analysis. This is here illustrated with one analysis of gelatinases by zymography. ${ }^{39}$ As is the case of in vitro tests, any in vivo test will have limitations. Coating with host molecules (eg, nanoparticle corona) may lead to immunological side effects in vivo. ${ }^{34}$ In contrast to organic biodegradable nanoparticles, including small virus particles, many nanoparticles with inorganic cores are not degradable, may persist and yield chronic deposition in tissues, sterile granuloma formation, and fibrosis. These aspects, and comparisons between degradable and nondegradable particles, may also be studied in the future by observations of air pouches after prolonged time intervals.

\section{Conclusion}

We conclude that standardized air pouch leukocytosis analysis fulfills the criteria as a test for innate immune functions and is also amenable for analysis of changes in adaptive immune functions, and we advocate the use of this analytical method. With microbial, pyrogenicity, 
cytotoxicity and in vitro immunological tests (eg, cytokine induction on specific cells) as first-line assays, the dermal air pouch analysis is - in addition to hematology parameters - a valuable tool to be implemented as second-line assay for all present and future nanomedicines. Experimental animal models for human disease are necessary for medical progress toward better diagnosis, prognoses, and therapies. Awareness of ethical issues has progressively diminished the use of mammalian vertebrates and led to a switch towards the use of frogs, zebrafish, worms, snails, and flies. Although ortholog models for many diseases already exist in these species, in particular for neurobiological research, for immunological evaluations and research such species replacement strategy is not broadly applicable. Because inflammation and adaptive immune mechanisms are increasingly being found to be important in tumor biology and nanoparticles are hailed as carriers for new cancer drugs, nanoparticle research for oncology applications needs to rely on evaluations by mammalian host experiments - such as the air pouch test. We recommend using only those nanoparticles parenterally (eg, for diagnostic purposes) that yield air pouch leukocytosis, equivalent to the negative PBS control.

\section{Acknowledgments}

The research leading to these results has received funding from the European Union Seventh Framework Programme (FP7/2007-2013) under grant agreement number 263307 and from The Fund for Scientific Research of Flanders (FWOVlaanderen) and the Geconcerteerde OnderzoeksActies GOA 2013014.

\section{Author contributions}

JV, CD, IVA, NB, IR, and GO optimized and standardized the air pouch test and performed enzymatic, cellular and cytometric analyses. LLI, IR, JK, JPL, SM, EL, MCF, MKA, LSA, and IL manufactured and characterized various nanoparticles. DRE, LS, and GO coordinated this study. All authors collaborated in the writing and approved the manuscript.

\section{Disclosure}

The authors declare no conflicts of interest in this work.

\section{References}

1. Seyle H. Effect of inflammation upon the growth of transplantable neoplasms as demonstrated by the double granuloma-pouch technique. Br J Cancer. 1957;11(4):550-553.

2. Hanahan D, Weinberg RA. Hallmarks of cancer: the next generation. Cell. 2011;144(5):646-674.
3. Taylor PE, Tejada C, Sanchez M. The effect of malnutrition on the inflammatory response as exhibited by the granuloma pouch of the rat. J Exp Med. 1967;126(4):539-556.

4. Folkman J, Merler E, Abernathy C, Williams G. Isolation of a tumor factor responsible for angiogenesis. J Exp Med. 1971;133(2):275-288.

5. Okudaira M, Schwarz J. Histoplasma capsulatum infection in rat air pouch. Arch Pathol. 1962;74:239-243.

6. Sedgwick AD, Sin YM, Edwards JC, Willoughby DA. Increased inflammatory reactivity in newly formed lining tissue. J Pathol. 1983; 141(4):483-495.

7. Dalhoff A, Frank G, Luckhaus G. The granuloma pouch: an in vivo model for pharmacokinetic and chemotherapeutic investigations. I. Biochemical and histological characterization. Infection. 1982; 10(6):354-360.

8. Konno S, Tsurufuji S. Induction of zymosan-air-pouch inflammation in rats and its characterization with reference to the effects of anticomplementary and anti-inflammatory agents. Br J Pharmacol. 1983;80(2):269-277.

9. Sedgwick AD, Lees P. A comparison of air pouch, sponge and pleurisy models of acute carrageenan inflammation in the rat. Agents Actions. 1986;18(3-4):439-446.

10. Balasubramaniam GS, Hurley JV. The structure and reactions of the microcirculation in a subcutaneous air pouch in the rat. J Pathol. 1987; 151(2):139-146.

11. Hambleton P, Miller P. Studies on immunological air pouch inflammation in the rat. Int Arch Allergy Appl Immunol. 1988;87(1):70-75.

12. Francis DJ, Forrest MJ, Brooks PM, Ghosh P. Retardation of articular cartilage degradation by glycosaminoglycan polysulfate, pentosan polysulfate, and DH-40J in the rat air pouch model. Arthritis Rheum. 1989;32(5):608-616.

13. Isaji M, Momose Y, Naito J. Enhancement of inflammatory reactions in a non-immunological air pouch model in rats. $B r J$ Exp Pathol. 1989;70(6):705-716.

14. Hashida R, Chiba K, Yamatsu I, Katayama K. Collagenase production in the exudate of a novel adjuvant-induced air pouch inflammation model in rats. Agents Actions. 1991;34(1-2):242-243.

15. Dawson J, Sedgwick AD, Edwards JC, Lees P. A comparative study of the cellular, exudative and histological responses to carrageenan, dextran and zymosan in the mouse. Int J Tissue React. 1991;13: 171-185.

16. Cronstein BN, Naime D, Ostad E. The antiinflammatory mechanism of methotrexate. Increased adenosine release at inflamed sites diminishes leukocyte accumulation in an in vivo model of inflammation. $J$ Clin Invest. 1993;92(6):2675-2682.

17. Vane JR, Mitchell JA, Appleton I, et al. Inducible isoforms of cyclooxygenase and nitric-oxide synthase in inflammation. Proc Natl Acad Sci. U S A. 1994;91(6):2046-2050.

18. Masferrer JL, Zweifel BS, Manning PT, et al. Selective inhibition of inducible cyclooxygenase 2 in vivo is antiinflammatory and nonulcerogenic. Proc Natl Acad Sci U SA. 1994;91(8):3228-3232.

19. Martin SW, Stevens AJ, Brennan BS, et al. Regional drug delivery I: permeability characteristics of the rat 6-day-old air pouch model of inflammation. Pharm Res. 1995;12(12):1980-1986.

20. Appleton I, Brown NJ, Willis D, et al. The role of vascular endothelial growth factor in a murine chronic granulomatous tissue air pouch model of angiogenesis. J Pathol. 1996;180(1):90-94.

21. Romano M, Faggioni R, Sironi M, et al. Carrageenan-induced acute inflammation in the mouse air pouch synovial model. Role of tumour necrosis factor. Mediators Inflamm. 1997;6(1):32-38.

22. Terkeltaub R, Baird S, Sears P, Santiago R, Boisvert W. The murine homolog of the interleukin-8 receptor CXCR-2 is essential for the occurrence of neutrophilic inflammation in the air pouch model of acute urate crystal-induced gouty synovitis. Arthritis Rheum. 1998;41(5): 900-909.

23. Smith CJ, Zhang Y, Koboldt CM, et al. Pharmacological analysis of cyclooxygenase-1 in inflammation. Proc Natl Acad Sci U S A. 1998; 95(22):13313-13318. 
24. Fernandez AA, Cavalcanti F, Miralles A, et al. Inhibition of cyclooxygenase-2 expression by 4-trifluoromethyl derivatives of salicylate, triflusal, and its deacetylated metabolite, 2-hydroxy-4trifluoromethylbenzoic acid. Mol Pharmacol. 1999;55(4):753-760.

25. Cronstein BN, Montesinos MC, Weissmann G. Salicylates and sulfasalazine, but not glucocorticoids, inhibit leukocyte accumulation by an adenosine-dependent mechanism that is independent of inhibition of prostaglandin synthesis and p105 of NFkappaB. Proc Natl Acad Sci US A. 1999;96(11):6377-6381.

26. Bertini R, Howard OM, Dong HF, et al. Thioredoxin, a redox enzyme released in infection and inflammation, is a unique chemoattractant for neutrophils, monocytes, and T cells. J Exp Med. 1999; 189(11):1783-1789.

27. Hooper KA, Nickolas TL, Yurkow EJ, Kohn J, Laskin DL. Characterization of the inflammatory response to biomaterials using a rodent air pouch model. J Biomed Mater Res. 2000;50(3):365-374.

28. Nakamura H, Herzenberg LA, Bai J, et al. Circulating thioredoxin suppresses lipopolysaccharide-induced neutrophil chemotaxis. Proc Natl Acad Sci U S A. 2001;98(26):15143-15148.

29. Hirasawa N, Ohtsu H, Watanabe T, Ohuchi K. Enhancement of neutrophil infiltration in histidine decarboxylase-deficient mice. Immunology. 2002;107(2):217-221.

30. Bouma G, Nikolic T, Coppens JM, et al. NOD mice have a severely impaired ability to recruit leukocytes into sites of inflammation. Eur J Immunol. 2005;35(1):225-235.

31. Merinen M, Irjala H, Salmi M, Jaakkola I, Hanninen A, Jalkanen S. Vascular adhesion protein-1 is involved in both acute and chronic inflammation in the mouse. Am J Pathol. 2005;166(3):793-800.

32. Delano DL, Montesinos MC, Desai A, et al. Genetically based resistance to the antiinflammatory effects of methotrexate in the air-pouch model of acute inflammation. Arthritis Rheum. 2005;52(8):2567-2575.

33. Teixeira CR, Teixeira MJ, Gomes RB, et al. Saliva from Lutzomyia longipalpis induces $\mathrm{CC}$ chemokine ligand 2/monocyte chemoattractant protein-1 expression and macrophage recruitment. J Immunol. 2005;175(12):8346-8353.
34. Oostingh GJ, Casals E, Italiani P, et al. Problems and challenges in the development and validation of human cell-based assays to determine nanoparticle-induced immunomodulatory effects. Part Fibre Toxicol. 2011;8(1):8.

35. Dobrovolskaia MA, McNeil SE. Immunological properties of engineered nanomaterials. Nat Nanotechnol. 2007;2(8):469-478.

36. Dobrovolskaia MA, Germolec DR, Weaver JL. Evaluation of nanoparticle immunotoxicity. Nat Nanotechnol. 2009;4(7):411-444.

37. Yoshida K, Koshino T, Saito T, Takagi T. Augmentation of anti-tumor effects of methotrexate by distilled water on Dunn osteosarcoma in mouse air pouch. Cancer Lett. 1998;126(2):193-197.

38. Piccard H, Berghmans N, Korpos E, et al. Glycosaminoglycan mimicry by COAM reduces melanoma growth through chemokine induction and function. Int J Cancer. 2012;131(4):E425-E436.

39. Vandooren J, Geurts N, Martens E, Van den Steen PE, Opdenakker G. Zymography methods for visualizing hydrolytic enzymes. Nat Methods. 2013;10(3):211-220.

40. Opdenakker G, Van den Steen PE, Van Damme J. Gelatinase B: a tuner and amplifier of immune functions. Trends Immunol. 2001;22(10): 571-579.

41. Descamps FJ, Martens E, Opdenakker G. Analysis of gelatinases in complex biological fluids and tissue extracts. Lab Invest. 2002;82(11): 1607-1608.

42. Vandooren J, Van den Steen PE, Opdenakker G. Biochemistry and molecular biology of gelatinase B or matrix metalloproteinase-9 (MMP-9): The next decade. Crit Rev Biochem Mol Biol. 2013;48(3): 222-272.

43. Li S, Starckx S, Martens E, et al. Myeloid cells are tunable by a polyanionic polysaccharide derivative and co-determine host rescue from lethal virus infection. J Leukoc Biol. 2010;88(5):1017-1029. 


\section{Supplementary materials}
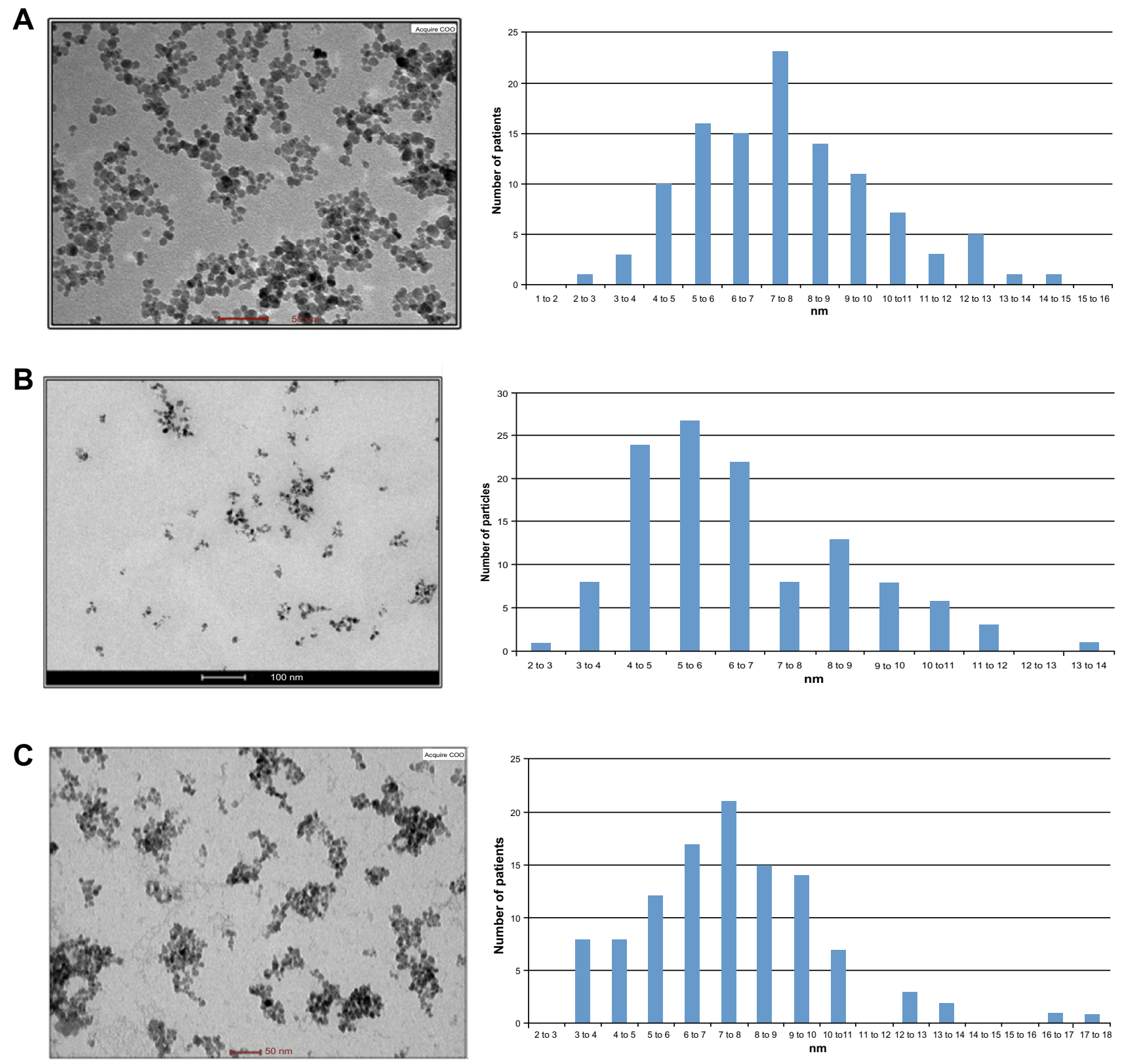

Figure SI CAN- $\gamma-\mathrm{Fe}_{2} \mathrm{O}_{3}$, type I PEI 25 - $\mathrm{CAN}-\gamma-\mathrm{Fe}_{2} \mathrm{O}_{3}$ and type II PEI ${ }_{25}-\mathrm{CAN}-\gamma-\mathrm{Fe}_{2} \mathrm{O}_{3} \mathrm{NPs}$ TEM and size distribution.

Notes: (A) CAN- $\gamma-\mathrm{Fe}_{2} \mathrm{O}_{3} \mathrm{NPs}$ (left) TEM microphotograph; (right) size distribution histogram of $7.6 \mathrm{I} \pm 2.33 \mathrm{~nm}$-sized CAN- $\gamma-\mathrm{Fe}_{2} \mathrm{O}_{3} \mathrm{NPs}$. (B) Type I PEI ${ }_{25}-\mathrm{CAN}-\gamma-\mathrm{Fe} \mathrm{O}_{2} \mathrm{O}_{3} \mathrm{NPs}$ (left) TEM microphotographs; (right) size distribution histogram of averaged 6.50 $\pm 2.15 \mathrm{~nm}$-sized Type I PEE ${ }_{25}-\mathrm{CAN}-\gamma-\mathrm{Fe}_{2} \mathrm{O}_{3} \mathrm{NPs}$. (C) Type II PEI ${ }_{8}-\mathrm{CAN}-\gamma-\mathrm{Fe}{ }_{2} \mathrm{O}_{3} \mathrm{NPs}$ (left) TEM microphotographs; (right) size distribution histogram of averaged $7.65 \pm 2.64 \mathrm{~nm}$-sized Type II PEI ${ }_{25}-\mathrm{CAN}-\gamma-\mathrm{Fe}_{2} \mathrm{O}_{3} \mathrm{NPs}^{2}$.

Abbreviations: NP, nanoparticle; PEl, polyethylene imine; TEM, transmission electron microscopy. 
A

DLS

Size distribution by intensity

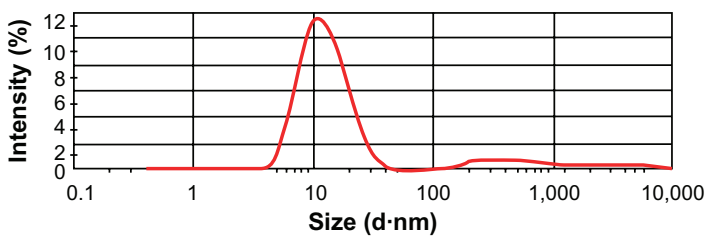

Average size $13 \pm 2.9 \mathrm{~nm}$
PDMAEMA SCPNs

\section{AFM}
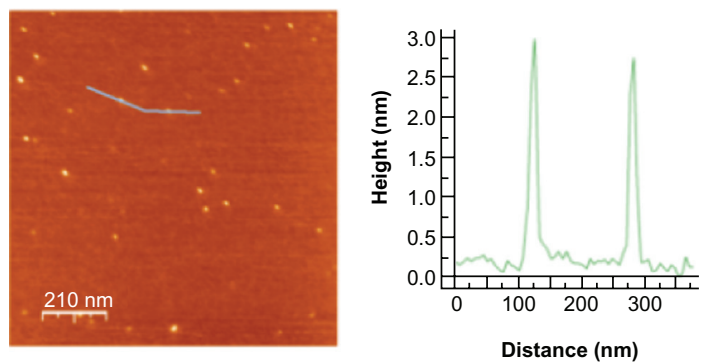

PMAAc SCPNs

B

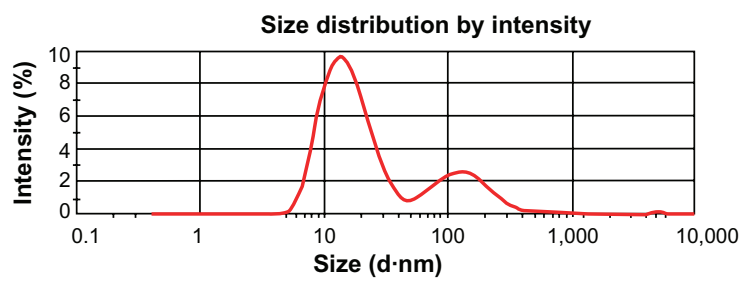

Average size $16.9 \pm 4.2 \mathrm{~nm}$
DLS

$600 \mathrm{~nm}$
AFM
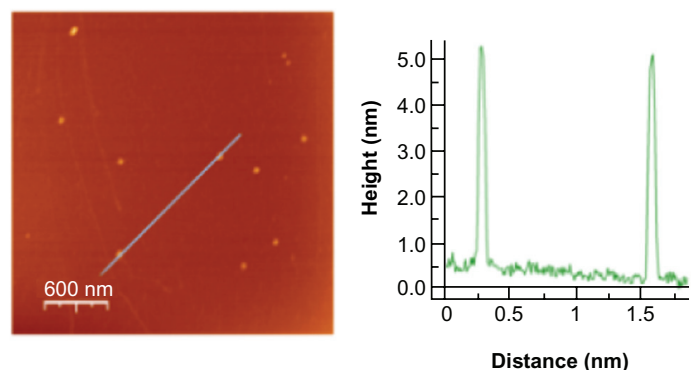

Figure S2 PDMAEMA and PMAAc SCPNs dynamic light scattering (DLS) and atomic force microscopy (AFM) images.

Notes: (A) PDMAEMA SCPNs (left) DLS measurement data: average size of I $3 \pm 2.9 \mathrm{~nm}$, and (right) AFM picture of dry nanoparticles around $3 \mathrm{~nm}$ in size; (B) PMAAc SCPNs (left) DLS measurement data: average size of $16 \pm 4.2 \mathrm{~nm}$; and (right) AFM picture of dry nanoparticles around $5 \mathrm{~nm}$ in size.

Abbreviations: PDMAEMA, poly(N,N-dimethylaminoethyl methacrylate); PMAAc, polymethacrylic acid; SCPN, single-chain polymeric nanoparticle.

A
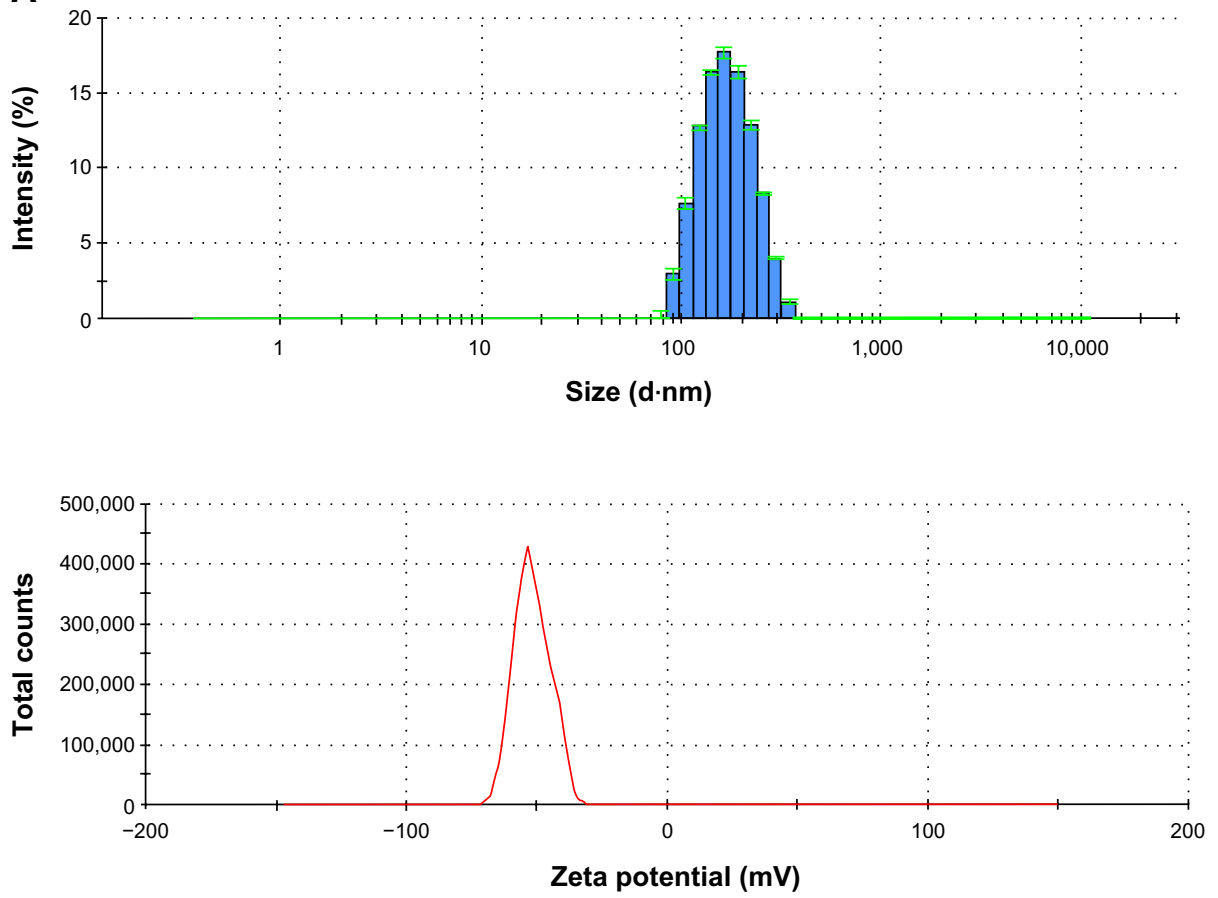

Figure S3 (Continued) 
B
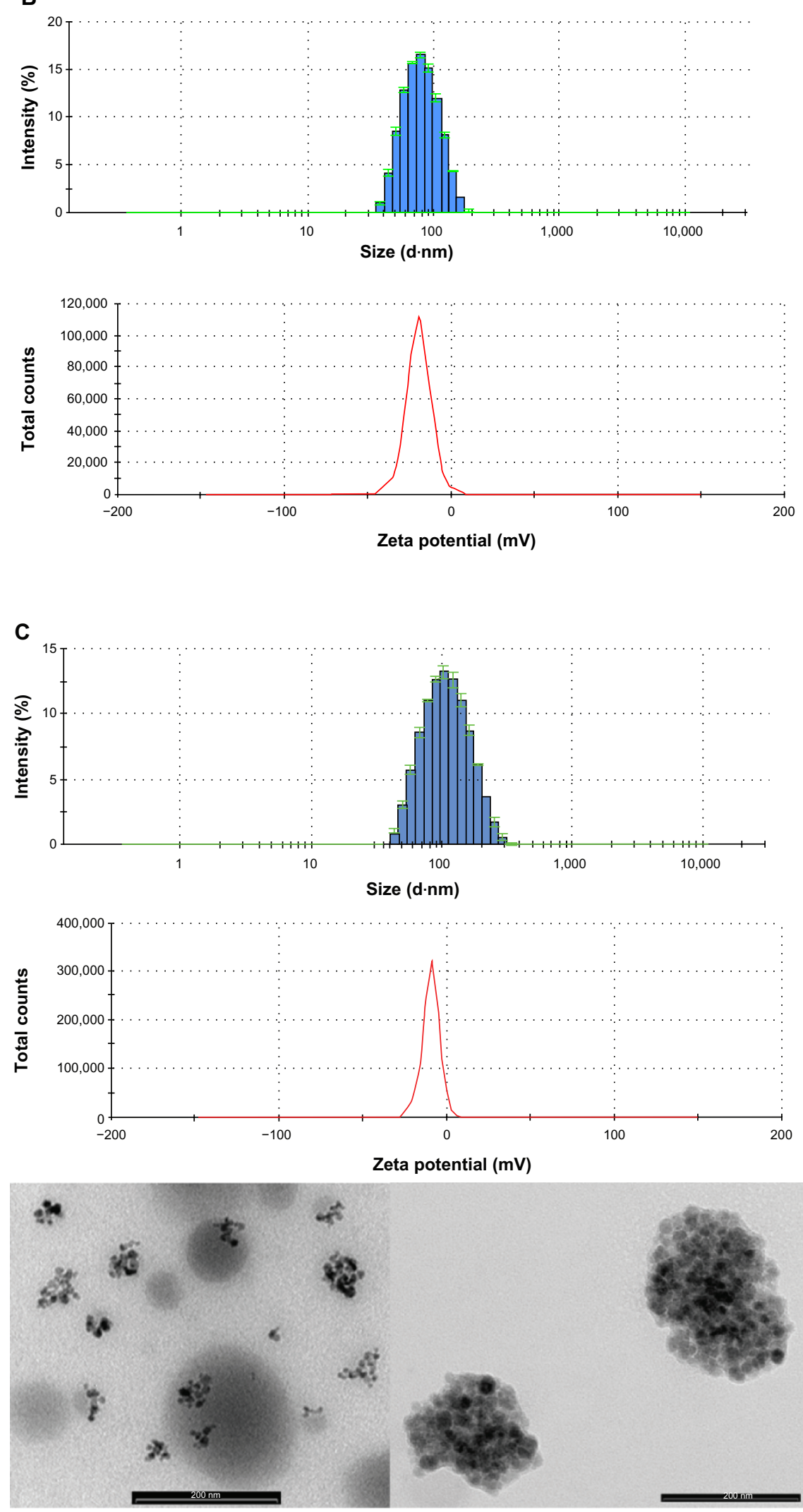

Figure S3 PLGA-COOH NPs, PLGA-b-PEG-COOH NPs and Magh@PNPs size distribution histograms, zeta potentials and transmission electron microscopy images. Notes: (A) PLGA-COOH NPs (top) size distribution histogram; (bottom) $\zeta$ potential of PLGA-COOH NPs; (B) PLGA-b-PEG-COOH NPs (top) size distribution histogram; (bottom) $\zeta$ potential of PLGA-b-PEG-COOH NPs; (C) Magh@PNPs (top) size distribution histogram, (middle) $\zeta$ potential, and (bottom) TEM microphotographs of Magh@PNPs. Abbreviations: NP, nanoparticle; PEG, polyethylene glycol; PLGA, poly(D,L-lactide-co-glycolide); PNPs, polymeric nanoparticles; TEM, transmission electron microscopy. 


\section{Publish your work in this journal}

The International Journal of Nanomedicine is an international, peerreviewed journal focusing on the application of nanotechnology in diagnostics, therapeutics, and drug delivery systems throughout the biomedical field. This journal is indexed on PubMed Central, MedLine, CAS, SciSearch $\AA$, Current Contents ${ }^{\circledR} /$ Clinical Medicine,

Journal Citation Reports/Science Edition, EMBase, Scopus and the Elsevier Bibliographic databases. The manuscript management system is completely online and includes a very quick and fair peer-review system, which is all easy to use. Visit http://www.dovepress.com/ testimonials.php to read real quotes from published authors.

Submit your manuscript here: http://www.dovepress.com/international-journal-of-nanomedicine-journal 